\title{
Modes de recrutement et de circulation des premiers membres britanniques et danois $d u$ Parlement européen
}

Patterns of Recruitment and Circulation among the First British and Danish Members of the European Parliament

Ann-Christina L. Knudsen

\section{(2) OpenEdition} Journals

\section{Édition électronique}

URL : http://journals.openedition.org/conflits/18329

DOI : $10.4000 /$ conflits. 18329

ISSN : $1777-5345$

\section{Éditeur :}

CCLS - Centre d'études sur les conflits lilberté et sécurité, L'Harmattan

\section{Édition imprimée}

Date de publication : 25 juin 2012

Pagination : 61-79

ISBN : 978-2-296-99408-9

ISSN : $1157-996 X$

\section{Référence électronique}

Ann-Christina L. Knudsen, « Modes de recrutement et de circulation des premiers membres britanniques et danois du Parlement européen », Cultures \& Conflits [En ligne], 85-86 | Printemps-Été 2012, mis en ligne le 25 juin 2013, consulté le 30 mars 2021. URL : http://journals.openedition.org/ conflits/18329; DOI : https://doi.org/10.4000/conflits.18329 


\section{Modes de recrutement et de circulation des premiers membres britanniques et danois du Parlement européen 1}

\section{Ann-Christina L. KNUDSEN}

Ann-Christina L. Knudsen est professeure à l'Institut pour la culture et la société de l'Université d'Aarbus (Danemark). Elle dirige actuellement un projet de recherche intitulé "Les institutions de la démocratie en transition. Les champs transnationaux dans la politique, l'administration et le droit après 1945 ». Elle a récemment publié : Farmers on Welfare. The Making of Europe's Common Agricultural Policy (Ithaca, Cornell University Press, 2009).

T es parlementaires sont à la fois les produits et les producteurs des instituـtions démocratiques. L'étude des modes de recrutement et des profils de carrière des parlementaires est donc une clé pour comprendre comment les élites politiques se constituent et comment la « nature » du politique change avec le temps ${ }^{2}$. Longtemps, les parlementaires ont eu pour « habitat » naturel le cadre national, et les études traitant du mode de recrutement et des parcours de carrière des parlementaires ont généralement été confinées à ce cadre. Pourtant, la période d'après-guerre se caractérise par une transnationalisation progressive de l'espace parlementaire, comme le montre Antonin Cohen dans sa contribution au présent numéro. Avec la création d'organisations internationales telles que la Communauté européenne du charbon et de l'acier, la Communauté économique européenne, le Conseil de l'Europe, l'Organisation du traité de l'Atlantique Nord (OTAN) et l'Union de l'Europe occidentale (UEO), un éventail d'assemblées parlementaires a en effet vu le jour. La représentation démocratique dans ces assemblées parlementaires a dès lors été instituée par le biais d'un double mandat, en vertu duquel les parlementaires étaient délégués par leurs parlements nationaux. Bien que les assem-

1. Texte traduit de l'anglais par Marie-Christine Boilard et révisé par la Rédaction de Cultures E Conflits.

2. Best H., Cotta M., "Elite Transformation and Modes of Representation since the MidNineteenth Century: Some Theoretical Reflections", in Best H., Cotta M. (eds.), Parliamentary Representatives in Europe 1848-2000. Legislative Recruitment and Careers in Eleven European Countries, Oxford, Oxford University Press, 2000, pp. 1-28. 
blées parlementaires aient été conçues pour n'avoir aucun ou seulement de très faibles pouvoirs législatifs, celles-ci ont néanmoins servi d'arènes politiques et sociales transnationales dont l'accès était exclusivement réservé aux parlementaires. Depuis les années 1940, des centaines de parlementaires provenant des États membres de ces différentes organisations ont ainsi détenu ce type de double mandat, et tous les grands partis et parlements nationaux se sont systématiquement impliqués dans les procédures de délégation qui en découlaient. Un fait particulièrement intéressant au sujet des agents sociaux détenteurs de ces doubles mandats est qu'ils pouvaient ainsi évoluer dans un espace parlementaire et politique traversant les frontières nationales et organisationnelles ${ }^{3}$.

Le Parlement européen (PE) a connu une trajectoire qualitativement différente des autres assemblées parlementaires mentionnées ci-dessus ${ }^{4}$. Cette différence peut être attribuée à l'institution d'une procédure électorale au suffrage universel direct à partir de 1979, ainsi qu'à l'introduction d'une série de pouvoirs formels supplémentaires visant à donner une légitimité démocratique au processus d'intégration européenne, tel que l'a montré Berthold Rittberger ${ }^{5}$. Mais, comme le souligne Antonin Cohen, elle est aussi le résultat de la progressive autonomisation et de la professionnalisation des parlementaires membres du Parlement européen (MPE). La littérature académique portant sur les carrières nationales et européennes des MPE est encore naissante et, lorsque de telles études existent, l'attention des chercheurs s'est principalement focalisée sur les MPE élus au suffrage universel direct ${ }^{6}$. Ces études ont néanmoins montré que, avec les parlementaires européens, parmi d'autres professions européennes, de nouveaux groupes sociaux ont ainsi émergé qui reliaient plusieurs champs de pouvoir à travers l'espace politique transnational. ${ }^{7}$ L'étude des pratiques de délégation antérieure à 1979 paraît donc d'autant plus nécessaire que les délégués reliaient entre elles des arènes politiques multiples, à la fois nationales et transnationales. Un aspect particulièrement

3. Guiraudon V., «L'espace socio-politique européen, un champ encore en friche ? », Cultures E Conflits, 38-39, 2000, pp. 7-38

4. Son nom était à l'origine l'Assemblé commune de la Communauté européenne du charbon et de l'acier. En 1962, l'Assemblée commune proclama qu'elle utiliserait désormais le terme de Parlement européen pour se désigner, mais ce dernier ne fut officiellement adopté que lors de l'Acte Unique européen de 1986. Le présent article fera usage du sigle PE.

5. Rittberger B., Building Europe's Parliament. Democratic Representation beyond the NationState, Oxford, Oxford University Press, 2005.

6. Voir par exemple Beauvallet W., «Institutionnalisation et professionnalisation de l'Europe politique, le cas des eurodéputés français ", Politique européenne, 9, 2003, pp. 99-122 ; Kirchner E., The European Parliament. Performance and Perspectives, Aldershot, Gower, 1984 ; Marrel G. et Payre R., « Des carrières au parlement : longévité des eurodéputés et institutionnalisation de l'arène parlementaire ", Politique européenne, 1-18, 2006, pp. 69-104 ; Scarrow S., "Political Career Paths and the European Parliament", Legislative Journal Quarterly, 22-2, 1997, pp. 253-263.

7. Kauppi N., "European Union Institutions and French Political Careers", Scandinavian Political Studies, 19-1, 1996, pp. 1-25 ; Georgakakis D. (ed.), Les métiers de l'Europe politique. Acteurs et professionnalisations de l'Union européenne, Strasbourg, Presses universitaires de Strasbourg, 2002. 
intéressant concernant les quelque $750 \mathrm{MPE}$ en fonction avant juin 1979 est en effet leur qualité d'agents multi-positionnés reliant entre eux les parlements nationaux et le $\mathrm{PE}$, ainsi que leurs groupes parlementaires et leurs partis politiques nationaux et ceux du PE.

Le PE des débuts est encore perçu comme un « cimetière d'éléphants ${ }^{8}$ ». Mais, dans les faits, il existe encore très peu de connaissances systématiques sur la façon dont le PE s'est institutionnalisé et inséré dans la transformation progressive des institutions démocratiques et des élites politiques. Le but de cet article est de contribuer à un renouvellement des travaux sur la façon dont les élites parlementaires se sont graduellement transformées avec l'institutionnalisation du PE, en examinant plus en détail le recrutement et les profils de carrière des MPE «double mandatés ». Dans cet article, je propose ainsi d'étudier les députés de deux pays ayant rejoint la Communauté européenne après le premier élargissement de 1973 : la Grande-Bretagne et le Danemark. Au total, 95 députés issus de ces deux États membres ont siégé au PE durant la période allant de janvier 1973 à juin 1979. Leur comparaison doit bien entendu tenir compte des similitudes et des différences structurelles dans la vie politique et parlementaire de ces deux pays, qui seront discutées plus en détail. D’un point de vue empirique, cet article repose sur une base de données contenant de nombreuses informations biographiques détaillant les profils sociaux des MPE, leur carrière politique, et en particulier leurs positions ministérielles avant et après avoir servi au PE, ainsi que la façon dont l'espace politique transnational est devenu partie intégrante de leur trajectoire et de leur carrière politique. Le choix de ces tard-venus à la Communauté peut également éclairer trois questions étroitement liées. Tout d'abord, l'analyse des parcours de carrière des MPE permet de montrer comment les parlementaires circulaient au sein et au-delà du cadre national. Ensuite, cette analyse peut nous renseigner sur la « réputation » du PE dans les années 1970, au moment où les pratiques de délégation étaient déjà bien institutionnalisées au sein des partis politiques et des parlements nationaux des six États fondateurs, et où le $\mathrm{PE}$ commençait à conquérir des pouvoirs plus formels. Enfin, cette analyse permet de comprendre comment les partis politiques commencèrent à gérer l'allocation des ressources à la suite de l'adhésion.

L'article se décompose de la manière suivante : la première section décrit d'abord le contexte et les principaux points de similitudes et de différences entre les systèmes politiques et parlementaires danois et britanniques dans les années 1970, ainsi que les changements clés qui se sont produits au sein du PE durant cette même période; dans les trois sections suivantes, les modes de recrutement et les tendances de circulation sont analysés à la lumière des profils sociaux et des parcours de carrière de ces deux populations de députés,

8. Verzichelli L., Vivere di politica. Come (non) cambiano le carriere politiche in Italia, Bologna, Il Mulino, 2010, p. 102. 
avant et après leur entrée au PE. Cette analyse inclut non seulement leur carrière nationale, mais également leurs mandats européens, c'est-à-dire leur activité au sein d'autres assemblées parlementaires que le PE, afin de mieux comprendre comment l'«Europe » est devenue partie intégrante des carrières parlementaires.

\section{ReMARQUeS DE MÉTHODE}

La base de données prosopographique sur laquelle repose cette analyse est constituée à partir de diverses sources. Les « listes grises " publiées par le PE avant la tenue de chaque session plénière ont été obtenues auprès du Centre archivistique et documentaire (CARDOC) au Luxembourg. Pour les députés britanniques, ces informations ont été complétées avec des informations biographiques tirées des sources suivantes : The Times Guide to the House of Commons, entre mai 1973 et juin 1979 ; The Times Guide to the European Parliament (1979) ; ainsi que les avis de décès parus dans les journaux britanniques suivants : The Independent, The Guardian, et The Daily Telegraph. En outre, certaines entrées ont été sélectionnées dans : Keith Laybourn, British Political Leaders. A Biographical Dictionary (Santa Barbara, ABC-Clio, 2001). Pour les députés danois, les informations ont été obtenues à partir d'entrées individuelles dans le Folketingets Håndbog, publié par le Folketingets Præsidium, Copenhague, Schultz Grafisk (plusieurs années) et Dansk Biografisk Leksikon, Copenhague, Gyldendal (1979). Dans certains cas, ces informations ont été complétées par d'autres sources disponibles en ligne, tels que des articles de journaux, la Welsh Biography Online, des descriptions de collections d'archives privées ou, dans de rares cas, les entrées de Wikipédia. De plus, des informations sur les MPE ont été obtenues à partir de : Sarah Charman et Keith Williams, The Parliamentarians' Role in the Alliance. The North Atlantic Assembly 1955-1980 (Assemblée de I'Atlantique Nord, Bruxelles, 1981), et de la liste en ligne des membres de l'Assemblée Parlementaire du Conseil de l'Europe depuis 1949.

\section{Trois parlements dans les années 1970}

Les élites politiques britanniques et danoises partagent l'expérience d'un long chemin vers l'adhésion à la Communauté qui débuta en 1961 avec leur première demande d'adhésion 9. La Grande-Bretagne et le Danemark avaient d'ores et déjà adhéré à d'autres organisations internationales dites occidentales, mais, dans le contexte de la Guerre froide, et alors que l'adhésion à l'OTAN avait provoqué maints désaccords politiques (tout en se heurtant à l'opposition populaire), la question de l'adhésion à la Communauté devint particulièrement épineuse. Cette opposition à l'adhésion communautaire traversait alors la plupart des partis politiques, mais la division était plus particulièrement forte au sein des partis se situant sur la gauche du spectre politique, tels que le Parti travailliste en Grande-Bretagne et le Parti social-démocrate au Danemark, profondément divisés sur le sujet. Une conséquence directe de ces divisions aura été le recours au référendum sur la question de l'adhésion, bien

9. Ludlow N. P., The European Community and the Crisis of the 1970s. Negotiating the Gaullist Challenge, London, Routledge, 2006 ; Olesen T. B., Den europaiske udfordring. EU, EØS og nordisk samarbejde i historisk belysning, Oslo, Europautredningen, 2011. 
que le contexte ayant conduit à en appeler au vote populaire ait été bien différent dans chaque pays. Au Danemark, le référendum eut lieu avant l'adhésion à la Communauté, le 2 octobre 1972. La Constitution de 1953 prévoyait en effet que toute participation à une coopération en matière de politique étrangère impliquant un abandon des pouvoirs constitutionnels dévolus au Parlement ne pourrait avoir lieu que si une majorité de plus de cinq-sixièmes des 179 membres du Parlement (MP) approuvait celui-ci. Ce qui signifiait que 150 députés devaient y être favorables. En revanche, il suffisait de réunir un tiers des parlementaires pour pouvoir recourir au référendum ${ }^{10}$. Quand le parlement passa au vote sur le traité d'adhésion, il manquait neuf voix au gouvernement social-démocrate alors au pouvoir, mais minoritaire, pour obtenir la majorité requise, rendant ainsi le référendum obligatoire, aux termes de la Constitution, pour poursuivre la procédure d'adhésion. Ceci eut lieu à peine une semaine après un premier référendum qui avait conduit au rejet de l'adhésion de la Norvège à la Communauté européenne, et tous les partis politiques danois, ainsi que les principales organisations syndicales et patronales se mobilisèrent dans la campagne ${ }^{11}$. Le résultat fut un taux de participation élevé de $90,1 \%$, mais, contrairement à leurs voisins norvégiens, 63,4\% des voix danoises se prononcèrent en faveur de l'adhésion ${ }^{12}$.

En Grande-Bretagne, la question de l'adhésion à la Communauté n'était pas l'unique sujet de division au sein de l'élite politique : le principe même du référendum était au cœur du débat ${ }^{13}$. Aucune disposition constitutionnelle ne prévoyait alors les conditions dans lesquelles il pouvait être procédé, ni à un abandon de souveraineté, ni à la tenue d'un référendum. La principale réticence à l'adhésion résultait de la forte croyance en la souveraineté du parlement, doublement mise à mal, toute mesure de démocratie directe en dehors des élections régulières étant considérée avec une forte dose de scepticisme ${ }^{14}$. Le recours au référendum fut donc une décision politique. L'adhésion avait été cimentée par un gouvernement conservateur dirigé par le Premier ministre (PM) Edward Heath, favorable à l'entrée de la Grande-Bretagne dans la Communauté européenne, et lorsque le Parti travailliste revint au pouvoir en octobre 1974 - après le fiasco d'un parlement sans majorité et deux séries de consultations électorales durant la même année -, il chercha à « renégocier » les termes de l'adhésion avec, pour exigence clé, la mise en place d'un mécanisme de correction visant à réduire la contribution financière du pays à la

10. Loi constitutionnelle du royaume du Danemark [Danmarks Riges Grundlov], 5 juin 1953, Sections 20 et 42. http://www.ft.dk/Dokumenter/Publikationer/Grundloven/ /media/Pdf_ materiale/Pdf_publikationer/Grundloven/Danmarks Riges Grundlov pdf.ashx (dernière consultation en août 2011).

11. Petersen N., Elklit J., "Denmark enters the European Community”, Scandinavian Political Studies, 8, 1973, pp. 198-213.

12. Knudsen A.-C. L., "Euroscepticism in Denmark", in Taggart P., Szczerbiak A. (eds.), Opposing Europe? The Comparative Party Politics of Euroscepticism, Oxford, Oxford University Press, 2008, vol. 1, pp. 152-167.

13. Butler D., Kitzinger U., The 1975 Referendum, Basingstoke, Macmillan, 1996.

14. Norris P., Electoral Change in Britain since 1945, Blackwell, Oxford, 1997, p. 10. 
Communauté ${ }^{15}$. Au vrai, les dirigeants du Parti travailliste étaient profondément divisés sur la question de l'adhésion, tant et si bien que son comité exécutif refusa d'envoyer des parlementaires au PE avant la tenue du référendum - les syndicats britanniques refusant, de leur côté, d'envoyer des délégués au Comité économique et social ${ }^{16}$. De surcroît, la crise économique généralisée du début des années 1970 avait conduit à un chômage important accompagné de grèves, en plus d'un tollé populaire contre la hausse des prix des denrées alimentaires, qui était largement attribuée à la politique agricole commune. Dans ce contexte, le PM Harold Wilson décida de soumettre l'adhésion au peuple, bien que les dirigeants du parti s'y soient précédemment opposé. Le référendum eut lieu le 5 juin 1975 et, malgré un taux de participation assez faible de $65 \%$, les résultats furent, eux, sans ambiguïté : les deux-tiers $(67,2 \%)$ des électeurs s'étant présentés aux urnes se prononcèrent en faveur du maintien de leur pays dans la Communauté. Le Parti travailliste décida alors de « normaliser» l'adhésion en envoyant, entre autres, ses délégués au PE.

D’une manière générale, les systèmes politiques britannique et danois se sont montrés très stables dans la période de l'après-guerre. La confiance du public dans le système électoral s'était traduite, entre autres choses, par un taux élevé de participation aux élections législatives, généralement compris entre $80 \%$ et $90 \%$. Des différences au niveau des structures de représentation politique nationale restent cependant à souligner. En Grande-Bretagne, les modes de recrutement et les parcours de carrière politique variaient selon la chambre. Les représentants à la Chambre des Communes étaient élus au suffrage universel, par un vote uninominal majoritaire à un tour. La Chambre des Lords, en revanche, était composée de pairs héréditaires issus de l'aristocratie, qui se trouvaient là en vertu du droit de naissance, mais également, à partir de 1958, de pairs nommés à vie par leur parti politique. Les MPE étaient issus de l'une de ces deux chambres, tout comme ils l'avaient été jusqu'alors dans les autres assemblées parlementaires européennes auxquelles la Grande-Bretagne participait. Au Danemark, le système parlementaire bicaméral avait été aboli avec la révision constitutionnelle de 1953, en faveur d'un parlement monocaméral, le Folketinget, élu au suffrage universel suivant le principe de la représentation proportionnelle dans les conscriptions locales.

En Grande-Bretagne, dans l'immédiat après-guerre, les principaux partis politiques, le Parti travailliste et le Parti conservateur, se relayaient pour diriger le pays à la tête du gouvernement. Au tournant de la fin des années 1960, néanmoins, le système de représentation politique passa d'un bipartisme « dominant » à ce qui s'apparentait désormais davantage à un bipartisme en « déclin 17 ». La tradition était alors d'organiser des élections générales avec un

15. Il y avait différents points de vue quant à savoir si cette renégociation était, en réalité, une renégociation entre les partis politiques britanniques, ou entre le gouvernement travailliste et ses partenaires européens.

16. Butler D., Kitzinger U., op.cit., pp. 21-22. 
intervalle de quatre ans maximum ${ }^{18}$ et, malgré ces changements, les deux grands partis politiques continuèrent à s'arroger environ $95 \%$ des sièges, le système uninominal majoritaire à un tour jouant largement en leur faveur. Les plus petits partis politiques, tel que le Parti libéral et le Parti national écossais, commencèrent à contester de manière plus systématique que par le passé la domination des deux grands partis, ce qui était clairement visible à l'échelle politique locale. Après 1974, toutes les circonscriptions à travers la GrandeBretagne étaient désormais convoitées par des candidats en provenance d'au moins trois partis politiques différents ${ }^{19}$. Certains des petits partis politiques ayant remporté des sièges à la Chambre des communes obtinrent ainsi une certaine représentation au sein du PE, comme le montre le tableau 1.

Au Danemark, en revanche, il y avait en général huit à dix partis politiques différents représentés au parlement et le gouvernement était formé par une coalition de partis, ou était simplement minoritaire ${ }^{20}$. La Constitution de 1953 exigeait que les élections législatives se tiennent à intervalle de quatre ans maximum, bien que la tradition de former des gouvernements minoritaires, dont le soutien parlementaire était fragile, signifiait, en pratique, que la tenue d'élections législatives était plus fréquente. Les années 1970 furent particulièrement changeantes et l'électorat était invité à recomposer le Folketinget en moyenne tous les deux ans ${ }^{21}$. Le scrutin de décembre 1973 est souvent désigné comme une «élection-séisme». Avec un taux de renouvellement de $44 \%$, il remodela largement le système de partis danois ${ }^{22}$. En outre, les quatre «vieux » partis politiques - les sociaux-démocrates, les libéraux, les conservateurs et les sociaux-libéraux - y perdirent un terrain considérable. Les vainqueurs en furent plusieurs nouveaux partis créés au début des années 1970 : les démocrates du centre, les chrétiens-démocrates et le Parti du progrès. Deux partis politiques plus anciens, le Parti communiste et le Parti georgiste, (re)gagnèrent également du terrain lors des élections de décembre 1973. Plusieurs de ces petits partis danois gagnèrent également une représentation au PE.

17. Norris P., op.cit., p. 62.

18. Dans les années 1970, les élections législatives eurent lieu, en Grande-Bretagne, en juin 1970, février 1974, octobre 1974 et mai 1979.

19. Norris P., op.cit., p. 47.

20. Pedersen M., "The Incremental Transformation of the Danish Legislative Elite: The Party System as Prime Mover", in Best H., Cotta M. (eds.), Parliamentary Representatives in Europe 1848-2000. Legislative Recruitment and Careers in Eleven European Countries, Oxford, Oxford University Press, 2000, p. 33. Pour une enquête plus étendue, voir Kjær U., Pedersen M., De danske folketingsmedlemmer. En parlamentarisk elite og dens rekruttering, cirkulation og transformation 1849-2001, Gylling, Aarhus Universitetsforlag, 2004.

21. Dans les années 1970, les élections générales eurent lieu, au Danemark, en septembre 1971, décembre 1973, janvier 1975, février 1977 et octobre 1979.

22. Lors de l'élection de 1971, le taux de remplacement fur relativement élevé, soit de 34\%. Pedersen M., “The Incremental Transformation of the Danish Legislative Elite: The Party System as Prime Mover", in Best H., Cotta M. (eds.), Parliamentary Representatives in Europe 1848-2000. Legislative Recruitment and Careers in Eleven European Countries, Oxford, Oxford University Press, 2000, p. 35. 

Tableau - Membres du Parlement européen britanniques et danois
de janvier 1973 à juin 1979 par parti politique

\begin{tabular}{|c|c|c|c|}
\hline \multicolumn{2}{|c|}{ Grande-Bretagne } & \multicolumn{2}{c|}{ Danemark } \\
\hline Partis politiques & $\begin{array}{c}\text { Nombre } \\
\text { de MPE }\end{array}$ & Partis politiques & $\begin{array}{c}\text { Nombre } \\
\text { de MPE }\end{array}$ \\
\hline $\begin{array}{c}\text { Parti travailliste } \\
\text { Labour }\end{array}$ & 27 & $\begin{array}{c}\text { Parti social-démocrate } \\
\text { Socialdemokratiet }\end{array}$ & 14 \\
\hline $\begin{array}{c}\text { Conservateurs } \\
\text { Conservatives }\end{array}$ & 31 & $\begin{array}{c}\text { Parti populaire conservateur } \\
\text { Konservative Folkeparti }\end{array}$ & 4 \\
\hline $\begin{array}{c}\text { Libéraux } \\
\text { Liberals }\end{array}$ & 2 & $\begin{array}{c}\text { Parti libéral } \\
\text { Venstre }\end{array}$ & 3 \\
\hline $\begin{array}{c}\text { Parti national écossais* } \\
\text { Scottish National Party }\end{array}$ & 1 & $\begin{array}{c}\text { Parti du progrès } \\
\text { Fremskridtspartiet }\end{array}$ & 3 \\
\hline Déclaré indépendant* & 2 & $\begin{array}{c}\text { Parti populaire socialiste } \\
\text { Socialistisk Folkeparti }\end{array}$ & 3 \\
\hline Total & 1 & $\begin{array}{c}\text { Parti social-libéral } \\
\text { Radikale Venstre }\end{array}$ & 2 \\
\hline Non considéré & 64 & $\begin{array}{c}\text { Démocrates du centre } \\
\text { Centrum-Demokraterne }\end{array}$ & 1 \\
\hline Retsforbundet & 31 \\
\hline
\end{tabular}

* En dehors des groupes politiques du PE

** Ce délégué a d'abord été membre du Parti social-démocrate, qu'il quitte pour former le parti des Démocrates du centre après les élections de décembre 1973. Il était MPE tout au long de cette période, et a officiellement représenté les deux partis. Dans la mesure où il n'a représenté les sociaux-démocrates que pour une très courte période, il n'est ici décompté que comme un membre de son nouveau parti.

Dans la mesure où la durée du mandat des MPE n'était pas déterminée avant la première élection directe de 1979 , la situation politique interne des États membres avait une incidence directe sur la composition et la stabilité de leurs délégations au PE. La durée moyenne d'un mandat pour un MPE britannique était alors de 35 mois, mais une différence importante reste cependant à souligner entre les partis politiques puisque le Parti travailliste ne commença à déléguer ses députés au PE qu’après le référendum de 1975. Au total, les MPE 
conservateurs britanniques pouvaient siéger au PE pour un maximum de 77 mois consécutifs. Cinq d'entre eux ont effectivement siégé sur toute la période et deux autres, pendant 70 mois ou plus, mais la durée moyenne du mandat effectif d'un MPE conservateur britannique était de 40 mois. Pour leur part, les députés du Parti travailliste pouvaient siéger au PE pour un maximum de 47 mois, et neuf d'entre eux atteignirent ce maximum. Cela signifie que, parmi les MPE britanniques, chaque député conservateur siégea en moyenne $52 \%$ du temps total possible, contre $63 \%$ pour chaque député travailliste. En revanche, la tenue d'élections législatives plus fréquentes et le taux de renouvellement particulièrement élevé des membres du Folketinget dans les années 1970 affectèrent la stabilité de l'offre de MPE danois à travers tous les partis politiques. La durée du mandat effectif des MPE danois était en moyenne de 24 mois. Un seul député conservateur danois siégea au PE pendant 77 mois, et un seul autre presque aussi longtemps, soit 73 mois. Bien qu'il soit hors du propos de cet article de discuter en profondeur comment les MPE utilisaient leur temps pendant leur service au PE, il est à noter que la durée du mandat d'un $\mathrm{MP}(\mathrm{E})$ est susceptible d'avoir une incidence sur sa capacité à s'impliquer dans les questions politiques.

Le PE a lui-même subi de nombreux changements dans le cours des années 1970. À la fin des années 1970, ses pouvoirs formels furent étendus à deux reprises par les traités budgétaires de 1970 et 1975, lui donnant un certain «pouvoir du portefeuille » et un meilleur contrôle sur la Commission que ce qui avait été initialement prévu par le Traité de Rome ${ }^{23}$. L'élargissement de 1973 a non seulement eu pour résultat un accroissement du nombre des membres de la plupart des groupes politiques existant au PE, mais également la création de deux nouveaux groupes : le groupe conservateur européen et le groupe communiste et apparentés. Il s'en suivit également une expansion des comités qui prirent en charge une série de nouveaux sujets politiques, à travers lesquels les MPE firent entendre leur voix et furent en mesure de fixer, en partie au moins, l'agenda de la Communauté, en matière de politique environnementale par exemple ${ }^{24}$. Finalement, la tenue d'élections au suffrage universel direct avait fait l'objet de requêtes continues de la part de certain MPE depuis les années 1950, mais ce ne fut pas avant septembre 1976 que le Conseil européen décida finalement d'accepter cette proposition ${ }^{25}$. Initialement prévue pour 1978, l'adoption de mesures adéquates accusa des retards et elle fut repoussée d'un an. Avec l'introduction des élections directes, le double mandat devint optionnel et il revenait désormais aux parlements nationaux et aux

23. Knudsen A.-C., “The 1970 and 1975 Budgetary Treaties. Enhancing the Democratic Architecture of the Community Institutions”, in Laursen F. (ed.), Designing the European Union. From Paris to Lisbon, Basingstoke, Macmillan, 2012 (sous presse).

24. Meyer J.-H., «L'européanisation de la politique environnementale dans les années 1970 », Vingtième siècle, Revue d'bistoire, 1-113, 2012, pp. 117-126.

25. Parlement Européen, "Towards direct elections to the European Parliament. Paper written to mark the 30th anniversary of direct elections (June 1979)", CARDOC Journals, 4, mars 2009. 
partis politiques de décider si leurs délégués devaient devenir des parlementaires professionnels à plein temps au sein du PE. Le double mandat, avant juin 1979, supposait que tous les députés devaient partager leurs responsabilités entre les cadres national et communautaire. Toutefois, la nature éminemment politique et controversée de la question de l'adhésion à la Communauté, en Grande-Bretagne tout comme au Danemark, ainsi que l'éventail grandissant des sujets politiques auxquels le PE prit part pendant les années 1970, se traduisit par un emploi du temps très chargé au courant de la décennie pour les députés prenant leur poste au sérieux ${ }^{26}$.

L'élargissement a également quelque peu affecté l'équilibre de genre au sein du PE. Entre 1952 et 1979, un total de 30 femmes ont en effet siégé au PE, soit environ $4 \%$ du nombre total de délégués ${ }^{27}$. Avant l'arrivée des communistes italiens en 1968, il y avait eu à peine dix femmes MPE. Au cours de la décennie suivante, les six États fondateurs ne désignèrent que dix autres femmes en tant que MPE. Les délégations britanniques et danoises y ajoutèrent encore dix femmes supplémentaires au total, sept Britanniques et trois Danoises. Les femmes constituaient donc respectivement $11 \%$ et $9 \%$ des délégués britanniques et danois de janvier 1973 à juin 1979. Bien que l'adhésion de la Grande-Bretagne et du Danemark ait ainsi permis une plus grande représentation des femmes au sein du PE que par le passé, ce dernier resta néanmoins majoritairement un monde d'hommes jusqu'aux élections directes de juin 1979. Ce n'est que plus tard que le PE devint l'une des assemblées les plus féminisées d'Europe ${ }^{28}$.

\section{D’où venaient les MPE ?}

«Un homme qui occupe une fonction qui pourrait mener quelque part est plus susceptible d'avoir des ambitions de carrière qu'un homme qui occupe une fonction qui ne mène nulle part ${ }^{29}$. » Tel est l'argument principal du politiste Joseph A. Schlesinger dans son étude séminale sur la structure des carrières politiques aux États-Unis. Cette représentation des hommes politiques en individus s'efforçant de conquérir des positions de pouvoir matérielles et symboliques dans les hiérarchies politiques, au sein du gouvernement, au sein $\mathrm{du}$ parlement ou au sein des partis politiques, est centrale dans la plupart des études portant sur le fonctionnement du marché de l'emploi dans le secteur politique. On peut donc s'attendre à ce que toutes les positions politiques

26. Concernant l'emploi du temps des MPE, voir le débat à la Chambre des Communes, 25 mars 1977: http://hansard.millbanksystems.com/commons/1977/mar/25/european-parliamentdirect-elections\#S5CV0928PO_19770325_HOC_156 (dernière consultation : 22 juillet 2010).

27. Liste des délégués (avant 17 juillet 1979), Centre archivistique et documentaire (CARDOC), Luxembourg.

28. Beauvallet W. et Michon S., «Les femmes élues au Parlement européen (1979-2004). Modes de recrutement et stratégies d'investissement ", Regards sociologiques, 27-28, 2004, pp. 70-84.

29. «A man in an office which may lead somewhere is more likely to have office ambitions than a man in an office which leads nowhere.» Joseph A. Schlesinger, Ambition and Politics. Political Careers in the United States, Chicago, Rand McNally \& Company, 1966, p. 8. 
aient la réputation d'être dignes ou indignes d'être poursuivies, déterminant leur attrait ou non pour les hommes politiques. Cette section examine plus en détail les caractéristiques propres aux MPE britanniques et danois entre janvier 1973 et juin 1979, et discute la validité de l'argument rationaliste de Schlesinger dans le cas de ces deux populations de parlementaires.

La structure des postes ministériels différait quelque peu en GrandeBretagne et au Danemark. En Grande-Bretagne, les postes ministériels étaient classés par ordre hiérarchique décroissant comme suit : secrétaires d'État, ministres d'État, sous-secrétaires d'État parlementaires et secrétaires privés parlementaires. Les deux premières catégories étaient considérées comme des ministres de rang supérieur et étaient membres du Cabinet, tandis que les sous-secrétaires d'État parlementaires étaient considérés comme des ministres de rang inférieur et ne siégeaient pas au Cabinet. Pour leur part, les secrétaires privés parlementaires étaient des postes liés aux positions ministérielles de haut rang, dans la mesure où les ministres de plein exercice étaient autorisés à nommer un MP de confiance pour agir en tant qu'agent de liaison avec la Chambre des Communes. Les ministres pouvaient être recrutés au sein des deux chambres, bien que cette pratique fût plus courante sous les gouvernements conservateurs que travaillistes. À mesure que les responsabilités du gouvernement britannique se sont accrues dans l'après-guerre, le nombre de positions ministérielles a augmenté, au point que, entre 1945 et 1997, $39 \%$ des députés conservateurs et $31 \%$ des députés travaillistes avaient exercé des fonctions ministérielles au moment où ils quittaient le Parlement ${ }^{30}$. La structure des gouvernements britanniques rendait néanmoins l'accès aux positions ministérielles plus difficile pour les membres des plus petits partis politiques. $\mathrm{Au}$ Danemark, le système était un peu plus simple, dans la mesure où tous les ministres étaient membres du Cabinet, et où les distinctions de rang ministériel n'existaient pas. Compte tenu de la tradition danoise de gouvernements minoritaires ou de coalition, les parlementaires issus de petits partis politiques pouvaient aussi accéder aux positions ministérielles. De plus, bien que le système hautement formalisé du « cabinet fantôme » tel qu'il existait en GrandeBretagne n'existait pas au Danemark, les partis politiques ont graduellement eu tendance à désigner des porte-parole pour chaque grande question politique. Dans l'analyse qui suit, seul le plus haut poste ministériel occupé par un parlementaire avant de devenir MPE est pris en compte.

Au moment de l'adhésion à la Communauté, le parlementaire britannique et danois moyen susceptible de devenir MPE était un homme, âgé de 49 ans, légèrement mieux instruit que ses collègues parlementaires nationaux. Parmi les délégués britanniques, $20 \%$ avaient fréquenté une des écoles dites de

30. Rush M. et Cromwell V., "Continuity and Change. Legislative Recruitment in the United Kingdom 1868-1999”, in Best H., Cotta M. (eds.), Parliamentary Representatives in Europe 1848-2000. Legislative Recruitment and Careers in Eleven European Countries, Oxford, Oxford University Press, 2000, p. 486. 
"Clarendon »- aussi connues en tant que public schools (c'est-à-dire des écoles privées), telles que Eton, Harrow et Charterhouse - ou autre pensionnat. Un délégué danois avait fréquenté Harrow, mais cela était plutôt inhabituel et il n'était pas de tradition chez les parlementaires danois de fréquenter ce type d'écoles d'élite. Même s'il est clair que la majorité des diplômés de l'université parmi les MPE étaient issus des sciences humaines et sociales ou des lettres très généralement définies, il n’existe pas de modèle univoque dans leurs origines scolaires et professionnelles. Les travaux sur le parcours des agents ayant pris part au processus d'intégration européenne continentale, à ses débuts, ont montré une forte influence des juristes ${ }^{31}$. Mais ce n'est pas le cas ici. Seulement $17 \%$ des MPE britanniques étaient titulaires d'un diplôme en droit, et deux uniquement au sein des MPE danois. D’autres caractéristiques de leur profil se démarquent. Alors que $23 \%$ des MPE britanniques avaient reçu une éducation militaire et avaient exercé dans l'armée - pendant la Seconde Guerre mondiale pour la plupart, et plusieurs d'entre eux étaient également décorés en tant que héros de guerre -, seuls deux MPE danois étaient dans le même cas. Il convient de noter qu'au Danemark, un pays à tradition neutraliste, il n'est pas de coutume de mettre en avant les antécédents militaires dans les notices biographiques dont on peut disposer. Près d'un tiers des députés danois avaient exercé dans le secteur de l'éducation, chiffre légèrement inférieur en Grande-Bretagne, 12 \%. Tandis que $19 \%$ des MPE britanniques avaient d'une manière ou d'une autre un antécédent dans le secteur privé des entreprises et des affaires, ce chiffre s'élevait jusqu'à $29 \%$ parmi les MPE Danois. Seuls quatre MPE peuvent être considérés comme ayant été ouvriers, soit deux pour chaque pays. Enfin, une MPE britannique se classait elle-même comme "femme au foyer ». Un autre fait notable chez les Danois est que la grande majorité d'entre eux était déjà engagée dans des activités à l'étranger, à travers des organisations internationales et transnationales, ou avait effectué un séjour d'étude plus ou moins long à l'étranger, et avait déjà eu un employeur au profil international, ce qui suggère que ces MPE parlaient une ou plusieurs langues étrangères non scandinaves (on peut aisément présupposer qu'ils avaient aussi une bonne compréhension des langues norvégienne et suédoise). Ce paramètre a sans doute joué de manière différente dans le contexte britannique, où l'élite politique était plus largement issue des hautes sphères de la société qu'au Danemark, et dans la mesure où la maîtrise des langues étrangères représentait un enjeu moindre pour des individus issus d'une région du monde dont la langue officielle était une langue dominante.

31. Voir : Cohen A., Vauchez A., «Les juristes et l'ordre politique européen », Critique internationale, 1-26, 2005, pp. 97-99. Il convient de noter qu'il y avait des différences importantes entre les diplômes de droit en Grande-Bretagne et au Danemark. En Grande-Bretagne, l'examen du barreau qui suivait l'obtention d'un diplôme universitaire était généralement la voie d'entrée dans les professions juridiques, tandis qu'au Danemark un diplôme en droit était toujours basé sur cinq années d'études universitaires en droit. 
Environ un tiers des $64 \mathrm{MPE}$ britanniques qui furent délégués au PE entre janvier 1973 et juin 1979 avait auparavant des postes dénotant un parcours de carrière ministérielle : huit d'entre eux avaient été ministres de plein exercice, et donc membre du gouvernement, tandis que 12 autres avaient été sous-secrétaires parlementaires. En y ajoutant les six députés ayant été secrétaires privés parlementaires - tous issus du Parti travailliste - la proportion des MPE britanniques ayant occupé une position ministérielle s'élevait donc à $40 \%$. Bien que les six secrétaires privés parlementaires provenaient tous du Parti travailliste, il ne semble pas y avoir eu de différence significative entre les pratiques des partis politiques quant au recrutement des MPE ayant précédemment exercé des fonctions ministérielles. En outre, plusieurs MP avaient occupé des postes parlementaires de haut rang avant leur entrée au PE, tels que celui de Lord Commissioner of the Treasury. Au moins 10 autres avaient occupé des postes au sein de leur parti, tels que celui de «ministre fantôme » ou de «whip». Dans l'échantillon danois, huit d'entre eux, soit un quart des MPE avaient occupé des postes de ministres avant d'être délégué au PE, et tous provenaient des quatre «vieux » partis politiques qui avaient été au pouvoir. En résumé, les partis politiques danois et britanniques déléguaient un grand nombre de parlementaires possédant de l'ancienneté et une expertise politique considérable, à un âge où l'on pouvait s'attendre à ce qu'ils soient politiquement très actifs.

\section{Et où sont-ils allés ?}

Les parcours de carrière des MPE permettent, enfin, d'illustrer comment, au fil du temps, les (Euro)élites circulaient dans le système politique. L'étude de ces parcours de carrière doit néanmoins être abordée avec une certaine prudence. Lors de l'analyse (ou de la composition) des biographies d'hommes politiques, il est souvent possible de détecter (ou de faire ressortir) un domaine de spécialisation dans leur parcours professionnel. C’est par exemple le cas du conservateur d'origine écossaise, Alexander Fletcher, «nommé soussecrétaire d'État pour l'Ecosse en mai 1979, après avoir été un porte-parole de l'Opposition sur les affaires écossaises $32 »-$ comme le mentionne The Times Guide to the House of Commons, un outil de référence clé en matière de biographie parlementaire en Grande-Bretagne. Fletcher avait brièvement été MPE entre 1976 et 1977, où il siégea au sein de la commission de la Politique régionale et des transports. Il semble probable que la promotion de Fletcher fût le double résultat de la décision des dirigeants du parti de faire usage de ses compétences dans les affaires régionales et écossaises, d'une part, et de son propre désir de développer une spécialisation en adéquation avec son lieu de naissance et de résidence, d'autre part. Toutefois, il est impossible de présupposer un lien de causalité directe entre la spécialisation politique de Fletcher et son parcours de carrière. Comme Pipa Norris et Joni Lovenduski le souli-

32. The Times, Guide to the House of Commons, 1979, London, Times Books, p. 104. 
gnent dans leur étude sur le recrutement et la carrière politique dans le système britannique : "En pratique, compte tenu des accidents de la vie politique, de nombreuses carrières sont loin d'être linéaires. Au cours de leur vie, les hommes politiques peuvent se déplacer latéralement, sauter une étape ou deux en cours de route, ou monter et descendre les postes, comme le jeu des serpents et des échelles. 33 " La même remarque est susceptible de s'appliquer aux parcours de carrière des parlementaires britanniques et danois ${ }^{34}$. Il est toutefois possible, avec un échantillon de $95 \mathrm{MPE}$, d'aller au-delà de l'étude singulière des parcours de carrière. Les biographies collectives et relationnelles seront plutôt utilisées pour caractériser les types de postes politiques que les MPE ont détenus après leur mandat au PE. Comme précédemment, seule la plus haute fonction politique occupée par un parlementaire est prise en compte dans ce qui suit.

La caractérisation du PE comme étant un « cimetière d'éléphants » ne peut être confirmée pour aucune des deux populations concernées. Six ou sept des 64 MPE britanniques semblaient approcher de leur retraite de la vie politique au moment où leur mandat au PE a pris fin et la plupart d'entre eux avaient eu ce qui pourrait être décrit comme une splendide carrière politique ascendante. Ces taux et tendances se retrouvent à l'identique dans le cas du Danemark. La première délégation danoise, au début de 1973, était composée de $10 \mathrm{MPE}$, dont pas moins de quatre peuvent être considérés comme des retraités - un en provenance de chacun des quatre « vieux » partis. Ces quatre individus avaient tous occupé des postes de ministre, et possédaient, de diverses manières, un capital symbolique et politique considérable à l'échelle politique nationale. Mais tous se sont finalement retirés de la vie politique suite aux résultats dramatiques des élections législatives de décembre 1973.

Pour ceux qui ne prirent pas leur retraite, mais qui continuèrent plutôt leur carrière en politique, les perspectives semblaient quelque peu différentes suivant le pays. Seuls $10 \%$ des MPE britanniques accédèrent à des postes de ministre de premier rang ou de sous-secrétaire parlementaire. Si l'on ajoute à cela la catégorie des secrétaires privés parlementaires, le taux peut être étiré à $16 \%$. La plupart des autres députés continuèrent néanmoins à circuler et à obtenir d'autres types de positions politiques après leur mandat au PE. Un exemple de mobilité professionnelle vers le haut, en termes de carrière poli-

33. «In practice, given the accidents of political life, many careers are far from linear. During their lifetime, politicians may transfer laterally, skip a step or two along the way, or move up and down offices, like a game of snakes and ladders. " Norris P., Lovenduski J., Political Recruitment. Gender, Race and Class in the British Parliament, Cambridge, Cambridge University Press, 1995, p. 1.

34. Pour une discussion détaillée des modes de recrutement sociaux et géographiques au Danemark, voir Pedersen M., “The Incremental Transformation of the Danish Legislative Elite: The Party System as Prime Mover”, in Best H., Cotta M. (eds.), Parliamentary Representatives in Europe 1848-2000. Legislative Recruitment and Careers in Eleven European Countries, Oxford, Oxford University Press, 2000, pp. 29-49. 
tique, est celui de Betty Boothroyd, qui fut MPE de 1975 à 1977. Elle fut par la suite élue membre du comité exécutif national du Parti travailliste, de 1981 à 1987, avant de devenir, en 1992, la première femme élue en tant que Présidente (Speaker) de la Chambre des communes, un poste qu'elle occupa jusqu'en 2000. Il convient également de rappeler que, pour une partie des représentants britanniques qui étaient pairs héréditaires ou à vie à la Chambre des Lords, l'avancement dans les hiérarchies politiques formelles n'était pas nécessairement un objectif.

Les chances d'avancement dans la politique danoise après un double mandat en tant que MPE étaient un peu plus élevées qu'en Grande-Bretagne, environ $23 \%$. La tendance danoise est incarnée par un petit groupe allant et venant entre les plus hautes fonctions ministérielles et le PE, au cours d'une période caractérisée par des fréquents changements de gouvernement. Ceci est particulièrement bien illustré par l'exemple du social-démocrate Ivar Nørgaard et du libéral Ove Guldberg, qui occupèrent tous deux diverses positions ministérielles à partir de la fin des années 1960. Nørgaard était ministre des Affaires économiques extérieures lors de l'adhésion du Danemark à la Communauté. Guldberg, quant à lui, prit part à la toute première délégation nationale danoise au PE. Suite aux élections législatives de décembre 1973, les sociaux-démocrates perdirent leur emprise sur le gouvernement et il revint à Guldberg d'occuper « à son tour » le poste de ministre des Affaires économiques extérieures. Nørgaard, de son côté, prit directement le chemin du PE, où il rejoignit le bureau du groupe socialiste, avant de devenir vice-président du PE en 1975. Peu de temps après le changement de gouvernement, en mars 1975, Guldberg fut de nouveau (r)envoyé au PE, tandis que Nørgaard revint dans l'arène politique nationale pour occuper une fois de plus le poste de ministre Affaires économiques extérieures et nordiques. Dans le même mouvement, Guldberg prit la position de Nørgaard en tant que vice-président du $\mathrm{PE}$, et cela même si les deux parlementaires provenaient de deux partis politiques différents.

La circulation des MPE dans les espaces politiques nationaux démontre que le capital politique acquis au PE était par la suite convertible à l'identique sur les marchés de l'emploi politique britannique et danois, sans doute plus au Danemark - un petit État avec une économie largement ouverte - qu'en Grande-Bretagne. En dehors de la période initiale pendant laquelle le Parti travailliste a bloqué le mécanisme de délégation au PE, avant le référendum de 1975, l'allocation des ressources par les partis politiques britanniques et danois, en termes de délégation des MP au PE, ne semble pas avoir été déterminée par leur position concernant l'adhésion à la Communauté. 


\section{L'Europe comme vocation}

Dans son étude sur les députés français de 1979 à 2004, Willy Beauvallet discute la façon dont les «Eurodéputés » commencèrent à vivre non pas « de » mais « pour » l'Europe - une inversion de sens se référant à l'observation faite par Max Weber sur la manière dont les hommes politiques qui commencèrent à vivre « pour » la politique devinrent des professionnels et vécurent « de » la politique ${ }^{35}$. Cette observation est également pertinente ici. Il est important de souligner en effet que ce n'est pas uniquement les nouvelles structures d'opportunités offertes à partir des élections directes de juin 1979 qui ouvrirent un nouveau parcours de carrières aux MP britanniques et danois, mais leur participation antérieure au travail des autres assemblées parlementaires qui leur offrit une plateforme de recrutement au sein du PE. L' « Europe » était devenue une vocation à temps plein ou partiel pour un nombre grandissant de parlementaires possédant une spécialisation dans la représentation démocratique transnationale. Pas moins de $60 \%$ des MPE britanniques et $35 \%$ des MPE danois entre janvier 1973 et juin 1979, avaient été ou devinrent délégués à l'Assemblée parlementaire du Conseil de l'Europe (APCE), l'Assemblée de l'Atlantique Nord (AAN), l'Assemblée de l'Union de l'Europe occidentale (pour les parlementaires britanniques) ou le Conseil nordique (pour les parlementaires danois). L'APCE, en particulier, servit de terrain d'entraînement pour $39 \%$ des MPE britanniques et un tiers des MPE danois. Ces chiffres suggèrent, d'une part, qu'il existait un nombre croissant de parlementaires en Grande-Bretagne et au Danemark qui commençaient à se spécialiser dans le travail parlementaire international ; et, d'autre part, que nombre de parlementaires qui étaient effectivement impliqués dans le travail parlementaire interet transnational devinrent, en quelque sorte, des généralistes couvrant à différents moments plusieurs types d'assemblées parlementaires.

Sur les 64 parlementaires britanniques qui siégèrent en tant que MPE au cours de cette première période, $20 \%$ décidèrent de se présenter comme candidat au PE lors des élections directes de 1979 ou plus tard. Aucun d'entre eux n'était issu du Parti travailliste, alors que dix conservateurs, soit environ un tiers d'entre eux, souhaitaient faire de l'« Europe » leur vocation, et plusieurs siégèrent pour plus d'un mandat. Le Parti conservateur semble par conséquent avoir eu une politique encourageant ceux ayant déjà accumulé des connaissances sur le fonctionnement du PE à se présenter de nouveau, ce parti ayant déjà investi d'importantes ressources dans la mise en place du groupe conservateur européen au PE. L' «Europe » devint également une affaire de famille pour trois d'entre eux, dans la mesure où deux fils et un mari de ces MPE décidèrent de se présenter comme candidat au PE. En outre, cinq des conserva-

35. Beauvallet W., « Profession : Eurodeputé. Les élus français au Parlement européen et l’institutionnalisation d'une nouvelle figure politique et élective (1979-2004) », Thèse, Université de Strasbourg 3, Robert Schuman, 2007. 
teurs ayant décidé de se présenter aux élections directes occupaient un siège à la Chambre des Lords, et trois d'entre eux étaient des pairs héréditaires qui ne s'étaient jamais présentés comme candidat aux élections, et pour qui l'«Europe » devint une opportunité d'entrer dans la compétition politique. $\mathrm{Au}$ contraire, les dirigeants du Parti travailliste semblent avoir empêché au moins une partie des premiers MPE de se présenter comme candidat en 1979. Il y avait une perception résolument négative du double mandat, ou « double jobbing » (comme on en vint à designer ces appartenances multiples), et aucun des MPE travaillistes de la première cohorte britannique ne prit part aux élections directes du PE. Il existe néanmoins des exemples de MPE travaillistes de cette première cohorte ayant profité de ces nouvelles structures d'opportunités. Pour quelqu'un comme Geoffrey de Freitas, l'entrée au PE se fit après une carrière qui incluait des positions en tant que président à la fois de l'APCE et de l'AAN. Un autre exemple est celui de John Leslie Prescott, qui avait été membre de l'APCE avant le PE, et qui devint par la suite un poids lourd dans la direction du parti. Avec le changement de direction au sein du Parti travailliste en 2007, Prescott décida de retourner à l'APCE ainsi que de devenir représentant à l'Assemblée de l'UEO.

Parmi les premiers MPE issus des petits partis politiques, plusieurs firent de l'«Europe » une vocation. Winnie Ewing avait été MPE pour le Parti national écossais depuis juillet 1975, avant de devenir MPE élue au suffrage universel direct pour la circonscription européenne des Highlands and Islands pour encore deux décennies (1979-1999). « Mme Ecosse », comme elle fut surnommée dans les milieux européens, deviendra membre du parlement écossais à partir de 1999, date à laquelle elle était devenue une telle institution dans la vie politique écossaise qu'on lui demanda de diriger la cérémonie d'ouverture du nouveau parlement national ${ }^{36}$. Bien que les deux libéraux de l'échantillon eurent l'ambition de faire de l'« Europe » leur vocation en 1979, aucun ne réussit. Lord Gladwyn fut MPE durant les 44 mois premiers mois suivant l'adhésion de son pays à la Communauté. Il avait auparavant acquis une expérience internationale considérable, remontant à ses années de service au ministère des Affaires étrangères britannique pendant la Seconde Guerre mondiale, au sein duquel il participa entre autres choses aux grandes conférences de Téhéran, Yalta et Postdam, et aux négociations secrètes entre les gouvernements français, israéliens et britanniques à propos de l'invasion du canal de Suez en $1956{ }^{37}$. Toutefois, cette expérience internationale ne lui permit pas de compenser le fait qu'il était de loin le membre le plus âgé de la cohorte des MPE britanniques «double mandatés » (il était né en 1900), ne s'était jamais porté candidat aux élections par le passé, et perdit tout simplement la campagne. L'autre candidat en liste pour les élections directes était Russell Johnston, un ancien du Parti libéral, entre autres choses président du Parti

36. The Herald Scotland, "Madame Ecosse”, 23 July 2001.

37. The Independent, “Obituary: Lord Gladwyn”, 26 October 1996. 
libéral écossais de 1970 à 1974. Lorsque le PE s'avéra ne pas être une option de carrière pour Johnston, ce dernier contribua à fonder le parti des libérauxdémocrates, dont il devint plus tard le chef adjoint. La vocation européenne en politique ne se flétrit cependant point pour Johnston. Il devint membre de l'APCE en 1985, le principal dirigeant de l'Alliance pour les Démocrates et Libéraux pour l'Europe à l'APCE, puis le président de 1999 à 2000, avant de se retirer en 2008.

Parmi le premier groupe de MPE danois se trouvait un futur commissaire européen, Poul Dalsager, et quatre futurs membres du PE élus au suffrage universel direct (13\%). Ce nombre relativement faible n'est pas étonnant étant donné le taux élevé de renouvellement du Folketinget durant cette période. Toutefois, les quatre députés qui restèrent au PE après 1979, le firent pour plusieurs mandats. Deux d'entre eux ont connu des carrières de ministres entre leurs mandats au PE, Anker Kofoed du Parti libéral et Erhard Jakobsen des Démocrates du centre, et les deux autres, Jørgen Nielsen Brøndlund des libéraux et Ib Christensen du Parti georgiste, auraient en toute vraisemblance quitté la vie politique plus tôt qu'ils ne quittèrent le PE. Christensen est un autre exemple de stratégie des petits partis. Le Parti géorgien était entré et sorti du parlement à maintes reprises dans la période de l'après-guerre, mais ne retrouva jamais de siège après 1981. Mais Christensen, un eurosceptique, rejoignit le Mouvement contre la Communauté européenne - un mouvement unidimensionnel à la fois quant aux enjeux (les questions européennes) et quant à sa représentation (le Parlement européen) - qui fut également la première organisation politique sans représentation au sein d'un parlement national. Paradoxalement, Christensen devint l'un des premiers véritables parlementaires danois à extraire sa vocation hors de la sphère nationale, pour poursuivre une carrière internationale en opposition à l'ensemble de l'establishment des élites européennes.

\section{Conclusion}

Cet article visait à explorer les pratiques de recrutement et de circulation des MPE britannique et danois, de janvier 1973 à juin 1979, et à plaider en faveur d'une analyse des parcours de carrière politique qui englobe et dépasse tout à la fois la sphère nationale. Cette étude montre en premier lieu que, en Grande-Bretagne comme au Danemark, les principaux partis politiques avaient pour objectif de déléguer des individus ayant de l'ancienneté et des expériences gouvernementales au PE. Ceci suggère, d'une part, que les partis politiques visaient à allouer des ressources au PE sous la forme de parlementaires expérimentés et, d'autre part, que le $\mathrm{PE}$ avait une réputation attractive. Il s'ensuit que l'image rétrospective du PE comme un « cimetière d'éléphants » n'est pas confirmée, au moins à en juger par la population britannique et danoise. Une meilleure façon de décrire le PE pour la période visée est celle d'un terrain de jeu pour parlementaires de sexe masculin. Quand bien même 
ces deux nouvelles délégations accrurent le nombre de femmes présentes au PE (au contraire des Irlandais), leur proportion globale au regard du nombre total de sièges disponibles était à ce stade, en effet, encore faible. En deuxième lieu, cette étude met en lumière plusieurs tendances quant aux perspectives de carrière des parlementaires ayant siégé au PE. Certains furent remis en circulation au sein du gouvernement et autres positions politiques de haut rang, bien que le taux de renouvellement élevé des parlementaires danois durant la période ait partiellement entravé ce processus. Dans le même temps, néanmoins, les structures d'opportunité de représentation parlementaire se sont accrues, et l' «Europe » est devenue une spécialisation pour un nombre croissant de parlementaires. L'APCE a notamment servi de terrain d'entraînement pour les MPE britanniques et danois et certains y sont demeurés - ainsi que dans d'autres assemblées parlementaires - au cours d'une carrière politique transnationale qui les amena à occuper des positions de pouvoir parlementaire éminentes en dehors de leur pays d'élection. À la fin des années 1970, au moment où les partis politiques se préparaient pour les premières élections directes au PE, l'«Europe » était déjà institutionnalisée dans les procédures de recrutement, l'allocation des ressources, ainsi que dans les perspectives de carrière de nombreux parlementaires. 